\title{
Primary leiomyosarcoma of the breast: A case report and review of literature
}

\author{
Sandhya B. $\cdot$ Vivek Babu $\cdot$ Parthasarathy G $\cdot$ Vikram Kate $\cdot$ N. Ananthakrishnan $\cdot$ R. Krishnan
}

Received: 17 January 2009 / Accepted: 25 January 2009

(C) Association of Surgeons of India 2010

\begin{abstract}
Leiomyosarcomas of the breast are rare tumours. Only 18 such cases have been reported in the literature so far. We describe herein a case of primary leiomyosarcoma of the breast in a 54-year-old woman whose preoperative clinical and cytological findings indicated a benign breast tumour. However, a core needle biopsy of the lesion showed malignant spindle cells without any ductal elements. Histopathological examination of the mastectomy specimen suggested a diagnosis of leiomyosarcoma, which was subsequently confirmed by immunohistochemical analysis. Primary leiomyosarcoma of the breast is very rare and is difficult to diagnose preoperatively as it needs immuno-histochemical staining. It is necessary to excise the tumour with sufficient margins to prevent local recurrence. The role of postoperative adjuvant chemotherapy is not well documented.
\end{abstract}

Keywords Breast $\cdot$ Stromal tumours $\cdot$ Phyllodes

Sandhya B. · V. Babu $\cdot$ Parthasarathy G. $\cdot$ V. Kate

N. Ananthakrishnan $\cdot$ R. Krishnan

Departments of Surgery and Pathology,

Jawaharlal Institute of Postgraduate Medical

Education and Research,

Pondicherry - 605 006, India

Parthasarathy G. ( $\square)$

E-mail: gpartha79@hotmail.com

\section{Introduction}

Stromal sarcomas of the breast constitute about $1 \%$ of all malignant tumours of the breast [1, 2]. Primary leiomyosarcoma of the breast comprises a rare type of stromal sarcoma of the breast. To our knowledge only 18 cases have been reported in literature. As the presentation of leiomyosarcoma of breast is similar to any other stromal tumour such as malignant phyllodes tumour, preoperative diagnosis of this condition is rarely possible especially since there is need for mitotic figure counting and immunohistochemical staining to confirm the diagnosis [3]. In this context, we report the clinical, histopathological and immunohistochemical characteristics of this rare tumour of the breast.

\section{Case history}

A 54-year-old perimenopausal nulliparous lady presented with a rapidly growing lump in her left breast of 3 months duration. Patient had history of pain in the swelling for 1 month but no nipple discharge. She had no other significant complaints.

On examination, the mass was firm and measured $8 \times 10$ $\mathrm{cm}$ in size, not fixed to deeper tissues. Skin over the swelling was tense, shiny and warm with a few dilated veins. Examination of the left axilla revealed a single $2 \times 1 \mathrm{~cm}$ lymph node. Right breast and axilla were clinically normal. Examination of the other systems was normal. Fine needle aspiration from the mass was reported as phyllodes tumour with a suspicion of malignancy. Core needle biopsy of the lesion was done which showed spindle cell tumour with a mitotic rate of 5/10 High Power Fields (HPFs). There was no evidence of ductal elements or normal breast tissue in the 
biopsy specimen and possibility of stromal sarcoma such as leiomyosarcoma was suggested. Other haematological, biochemical and radiological investigations were within normal limits. The patient underwent a total mastectomy with level II axillary dissection.

\section{Pathology}

Gross examination revealed a gray-white, fleshy soft to firm tumour measuring $7 \times 7 \mathrm{~cm}$ situated just beneath the nipple. The tumour was well circumscribed with a satellite nodule and showed chalky areas of necrosis. The resected margins were grossly uninvolved. Axillary pad of fat revealed 15 lymph nodes ranging from 1 to $2 \mathrm{~cm}$ in diameter. Haematoxylin and Eosin stained sections, 10 in all, obtained by extensive sampling of the specimen showed a spindle cell tumour with moderate pleomorphism. On high power magnification, the nuclei were elongated with blunt ends and irregular nuclear contours; there were occasional prominent nucleoli and a mitotic rate of 6/10 HPFs (Fig. 1). The tumour showed focal myxoid change and necrosis. There were no ductal structures within the tumour (Fig. 2). Surrounding breast tissue showed fibrocystic change with extensive stromal hyalinisation and calcification. Resected margins were free of tumour and 15 lymph nodes identified in the axillary pad revealed reactive hyperplasia but no metastasis. With all these features, the possibility of a stromal sarcoma, distinct from phyllodes tumour, was considered. The differential diagnosis included leiomyosarcoma, fibrosarcoma, and malignant fibrous histiocytoma. Because of the typical appearance of the nuclei, leiomyosarcoma of the breast was favoured as the first diagnosis. Immunohistochemical staining was performed to confirm the diagnosis, which showed cytoplasmic positivity with desmin and smooth

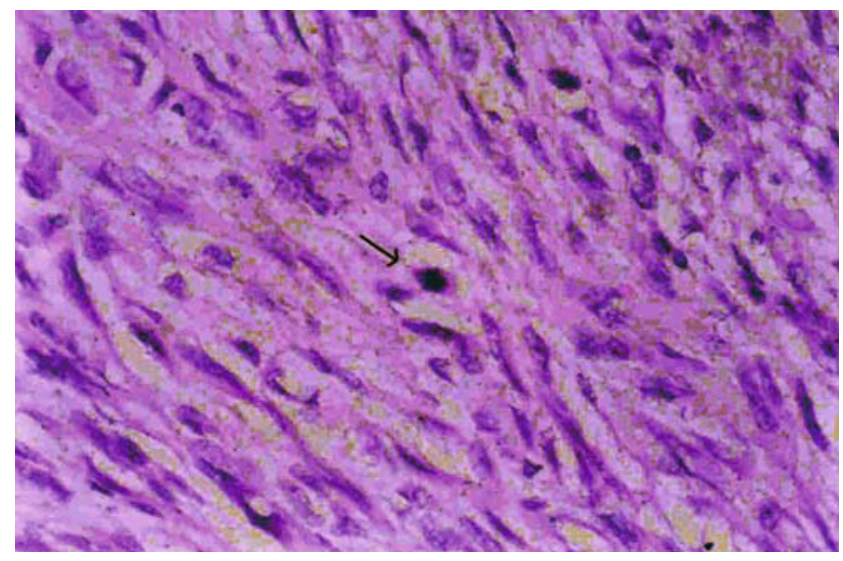

Fig. 1 Photomicrograph showing spindle shaped tumour cells with eosinophilic cytoplasm and blunt-ended nuclei. Note abnormal mitosis (arrow) (Haematoxylin and Eosin; $\times 400)$ muscle actin (SMA) within the tumour cells (Fig. 3). The tumour cells were negative for epithelial markers like cytokeratin and epithelial membrane antigen.

The patient was not given any postoperative chemotherapy. At 1 year follow up there was no evidence of locoregional or distant metastases.

\section{Discussion}

Stromal sarcoma is the generic term given to malignant breast tumours that arise from the specialised stroma of this organ but which lack the epithelial component of phyllodes tumour [4]. Histologically, most often these are fibrosarcoma, but may also be leiomyosarcoma, liposarcoma, rhabdomyosarcoma or malignant fibrous histiocytoma. Carcinoma with sarcomatoid features, also known as metaplastic carcinoma is an important differential diagnosis for these tumours. Breast sarcomas account for about $1 \%$ of all malignant breast tumours of which leiomyosarcoma is a rare entity $[1,2]$. This tumour can present either as a primary or a metastatic lesion in the breast. Metastasis to the breast is rare, with only four cases published in the literature to date $[5,6]$. Primary leiomyosarcoma of the breast is also a very rare tumour and less than 20 well-documented cases have been reported in literature [1]. It presents as a firm lobulated mass and resembles clinically a malignant phyllodes tumour. It is difficult to establish a diagnosis of this rare tumour by fine needle aspiration cytology $[7,8]$. Conventionally, the diagnosis of leiomyosarcoma of the breast is made on postoperative specimens by using immunohistochemical stains. The malignant spindle cells of the tumour are immunoreactive for SMA, vimentin and desmin and negative for epithelial markers and growth factor receptors [7]. In the present case too, smooth muscle markers like SMA and desmin were positive while cytokeratin and

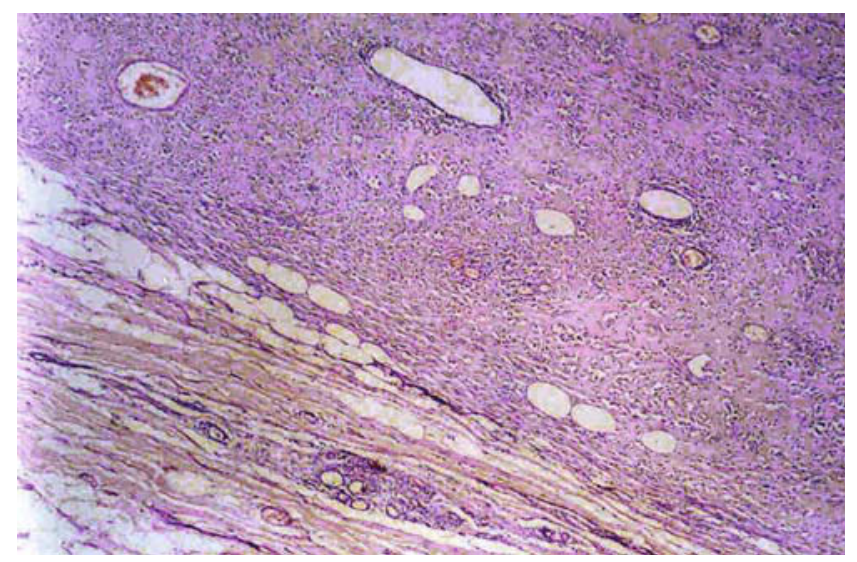

Fig. 2 Photomicrograph showing circumscribed stromal tumour with a pseudocapsule containing a breast lobule. Note absence of ductal elements within the tumour (Haematoxylin and Eosin; $\times 40)$ 


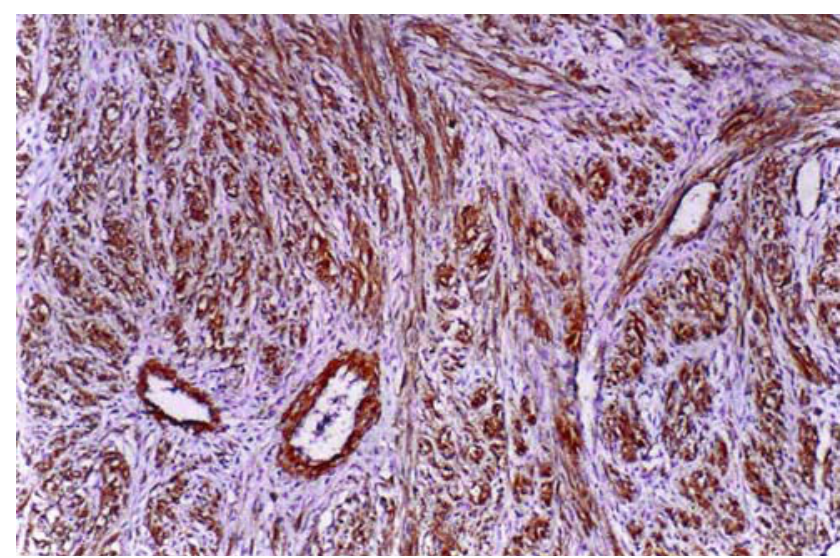

Fig. 3 Photomicrograph showing tumour cells with positivity for smooth muscle actin (Immunohistochemistry - Diaminobenzidine; $\times 100)$

epithelial membrane antigen were negative. This helped us to confirm that the tumour was indeed a leiomyosarcoma and not metaplastic carcinoma with sarcomatoid features or any of the other stromal sarcomas like fibrosarcoma or malignant fibrous histiocytoma. Phyllodes tumour of the breast may occasionally have a very prominent stromal component with smooth muscle differentiation and scanty epithelial elements. However, even after extensive sampling we failed to demonstrate any epithelial elements. This helped us in ruling out the possibility of phyllodes tumour with smooth muscle differentiation.

When a diagnosis of leiomyosarcoma is made either preoperatively or at the time of surgery an extended excision with adequate margins should be performed as it is a locally invasive neoplasm [3]. As only a few cases have been described in literature so far, a general consensus on the treatment of this tumour is still lacking. Surgery is the mainstay of treatment and the role of postoperative chemotherapy is doubtful. There is no definite evidence to suggest that chemotherapy would be of any benefit since metastasis might appear decades later without any treatment [2]. In the case presented above, since the surgical resected margins were free of tumour, no further therapy was administrated and after 1 year of follow up, the patient has not shown any evidence of recurrence. Axillary block dissection is usually not recommended, since none of the reported cases have shown nodal metastases. In the present case, however, as the preoperative diagnosis had not been confirmed, axillary lymph node dissection was also undertaken.

\section{References}

1. Liang WC, Sickle-Santanello BJ, Nims TA, Accelta PA (2003) Primary leiomyosarcoma of the breast: A case report with review of the literature. Breast J 9:494-496

2. Ugras S, Dilek ON, Karaayvaz M, Dilek H, Peter O, Barut I (1997) Primary leiomyosarcoma of the breast. Surg Today 27:1082-1085

3. Shinto O, Yashiro M, Yamada N, Matsuoka T, Ohira M, Ishikawa T, et al. (2002) Primary leiomyosarcoma of the breast: Report of a case. Surg Today 32:716-719

4. Rosai J (1996) Breast. In: Ackerman's Surgical Pathology. Rosai J (Ed.), 8th edtion, New York, Mosby; pp 1565-1661

5. Madigan MN, Dempsey PJ, Krishnamurthy S (2003) Ultrasound-guided fine needle aspiration cytodiagnosis of leiomyosarcoma metastatic to the breast. A case report. Acta Cytol 47:783-786

6. Lin $\mathrm{CH}$, Yeh CN, Chen MF (2003) Breast metastasis from uterine leiomyosarcoma: A case report. Arch Gynecol Obstet 267:233-235

7. Szekely E, Madaras L, Kulka J, Jaray B, Nagy L (2001) Leiomyosarcoma of the female breast. Pathol Oncol Res 7:151-153

8. Jun Wei X, Hiotis K, Garcia R, Hummel Levine P (2003) Leiomyosarcoma of the breast: $\mathrm{D}$ difficult diagnosis on fine needle aspiration biopsy. Diagn Cytopathol 29:172-178 